\title{
EVALUATION OF NEUROGENIC AND HUMORAL FACTORS IN BLOOD PRESSURE MAINTENANCE IN NORMAL AND TOXEMIC PREGNANCY USING TETRAETHYLAMMONIUM CHLORIDE
}

\author{
By ALBERT A. BRUST, N. S. ASSALI, ANd EUGENE B. FERRIS \\ (From the Departments of Internal Medicine and Obstetrics, College of Medicine, University \\ of Cincinnati, and the Cincinnati General Hospital, Cincinnati)
}

(Received for publication July 6, 1948)

Elevation of the arterial blood pressure is one of the most characteristic signs of toxemia of pregnancy. That this blood pressure elevation must reflect an increase in peripheral resistance in the toxemic patient is well recognized. More and more emphasis has been placed on the observation of vasoconstrictor phenomena in this illness so that arteriolar spasm is now thought to represent a frequent if not universal concomitant of toxemia. Since, however, it is well established that constrictor responses may be initiated through both neurogenic and humoral mechanisms, the question as to the primacy of either of these factors in the hypertension of toxemia remains unanswered.

Designation of the specific hypertensive disease of pregnancy as toxemia implies a primary humoral disorder. Despite the lack of conclusive evidence to support this concept, it has gained wide acceptance. Investigators have been occupied with the search for hormones, pressor agents and enzyme inhibitors peculiar to gestation. Although significant discoveries have been made, none has clarified the nature of blood pressure control in either toxemia or normal pregnancy.

The recent development of pharmacologic agents which induce transient paralysis of the autonomic nervous system has provided a valuable adjunct for study of the mechanisms involved in blood pressure control. Tetraethylammonium chloride (TEAC), by blocking sympathetic impulse transmission at the ganglionic level, abolishes neurogenic tone. Failure to obtain a fall in blood pressure following TEAC suggests the activity of humoral agents. After sympathetic block, the blood pressure remains responsive to pressor substances and thus must be maintained by humoral mechanisms together with intrinsic vascular tone. Accordingly, an assay of TEAC responses in normal and toxemic pregnancy was undertaken in an effort to throw light on the relative importance of humoral and neurogenic factors in blood pressure maintenance in these conditions.

\section{MATERIAL AND METHODS}

Forty-three patients were studied, including the following groups: normal non-pregnant controls, 10 subjects; normal term pregnancy, 10 patients; and toxemia of pregnancy, 23 patients. In the toxemic group, 12 patients were designated as having preeclampsia, five eclampsia, and six had toxemia superimposed on an antecedent hypertension. The criteria for classification of the above patients are given at the end of this section.

TEAC 1 studies were performed on all pregnant patients in both the prepartum and postpartum periods. Prepartum responses of normal pregnancies were recorded as near term as possible. Following delivery the same patients were tested again, in every case between 24 and 48 hours postpartum.

In the toxemia group, antepartum TEAC responses were recorded at the time when clinical signs and symptoms of toxemia were most prominent and prior to the institution of therapy. After parturition, TEAC responses were observed on one or more occasions between the second and seventh postpartum day. In all cases it was attempted to secure responses coincident with the recovery from toxemia as determined by clinical and laboratory evidence. In addition, 12 patients were tested repeatedly during the ninth month of pregnancy and the puerperal period at times when the clinical course suggested either abatement or increased severity of the toxemic state. Responses were also recorded on these patients at intervals up to six months postpartum for control purposes.

Normal non-pregnant women, utilized in this study as a control group, were subjected to a single test of blood pressure response following TEAC.

All studies were conducted with the patient in the supine position. After a control period during which the blood pressure was allowed to stabilize, $4 \mathrm{cc}$. of TEAC (400 mg.) were injected intravenously to accomplish autonomic blockade. Following the injection the blood pressure was recorded (sphygmomanometer) at half-minute intervals for six minutes and at oneminute intervals for 10 to 30 minutes thereafter. In this

1 Etamon chloride supplied by Parke, Davis and Company, through the courtesy of Dr. E. C. Vonder Heide. 
study the control blood pressure represents the mean of five or more readings following stabilization of the pressure in the recumbent position. The lowest point to which the pressure descended in the first five minutes following injection has been termed the TEAC floor.

Criteria for the diagnostic classification of the various groups studied were as follows:

Normal non-pregnant controls: These included healthy nulliparous females from the hospital staff and medical students. Their ages ranged from 22 to 37 years. None gave historical or present evidence of hypertension.

Normal term pregnancy: Included in this group were four primiparae and six multiparae who had been under observation in the prenatal clinic. Their ages ranged from 16 to 36 years. None had evidence of present or past hypertension, or previous toxemia.

Toxemias of pregnancy: Hypertension, albuminuria and edema occurring in the third trimester were used as the prime differential diagnostic points of this group. Subjective symptoms (headache, epigastric pain, scotomata) were given only secondary consideration. The ages of the 23 toxemic patients ranged from 14 to 42 years, with a mean of 25 years.

Twelve of the 23 patients in this group were considered to be preeclamptic. Nine were primiparae and three multiparae. From the standpoint of clinical severity, three patients had Grade I and nine patients Grade II preeclampsia. Clinical and laboratory examinations during hospitalization together with observations for four to six months postpartum served to confirm the diagnosis. The majority of patients, however, showed albuminuria and hypertension of such degree as to leave no question as to clarity of the diagnosis at the time of confinement.
Convulsions were observed in five patients (three primiparae, two multiparae) and these have been classified as eclampsia. The seizures occurred postpartum in two patients who had been considered preeclamptic prior to delivery.

In six patients who presented the classical toxemia triad there was adequate evidence of pre-pregnant hypertension. Of these, two were primiparae and four multiparae. Previous blood pressure observations and urinalyses were available from hospital records to indicate a further elevation of the hypertension and the appearance of albuminuria with relation to the current pregnancy. In addition, a three to six month follow-up of these patients following delivery has provided further evidence of the accuracy of the diagnosis at the time of hospitalization.

\section{RESULTS}

The TEAC blood pressure response and floor deviate strikingly and consistently from the normal, both in toxemia of pregnancy and in normal pregnancy at term. In toxemia, the floor is consistently elevated and falls to normal after delivery and recovery from toxemia. In normal term pregnancy, the floor is consistently depressed and rises to normal following parturition.

Normal non-pregnant controls: The results are indicated in Figure 1. TEAC induces very little fall in blood pressure. The mean control blood pressure for the group was $112 / 72 \mathrm{~mm}$. $\mathrm{Hg}$, and the mean TEAC floor was $103 / 67 \mathrm{~mm}$. $\mathrm{Hg}$. The

TEAC RESPONSES IN NORMAL NON-PREGNANT FEMALES

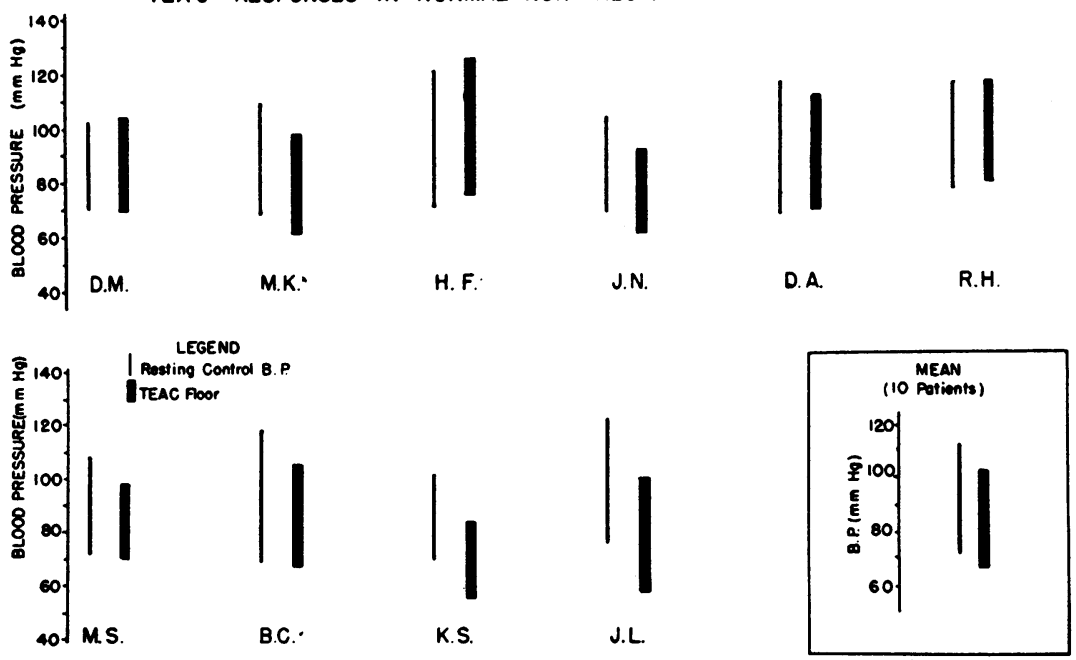

Fig. 1. Blood Pressure. Responses of Normal Non-Pregnant Females Following AUtonomic Blockade With TEAC

The thin vertical line represents the control blood pressure and the heavier line the TEAC floor. At the lower right is depicted the mean group response. Note the meager magnitude of the response $(10 / 5 \mathrm{~mm} . \mathrm{Hg})$. 


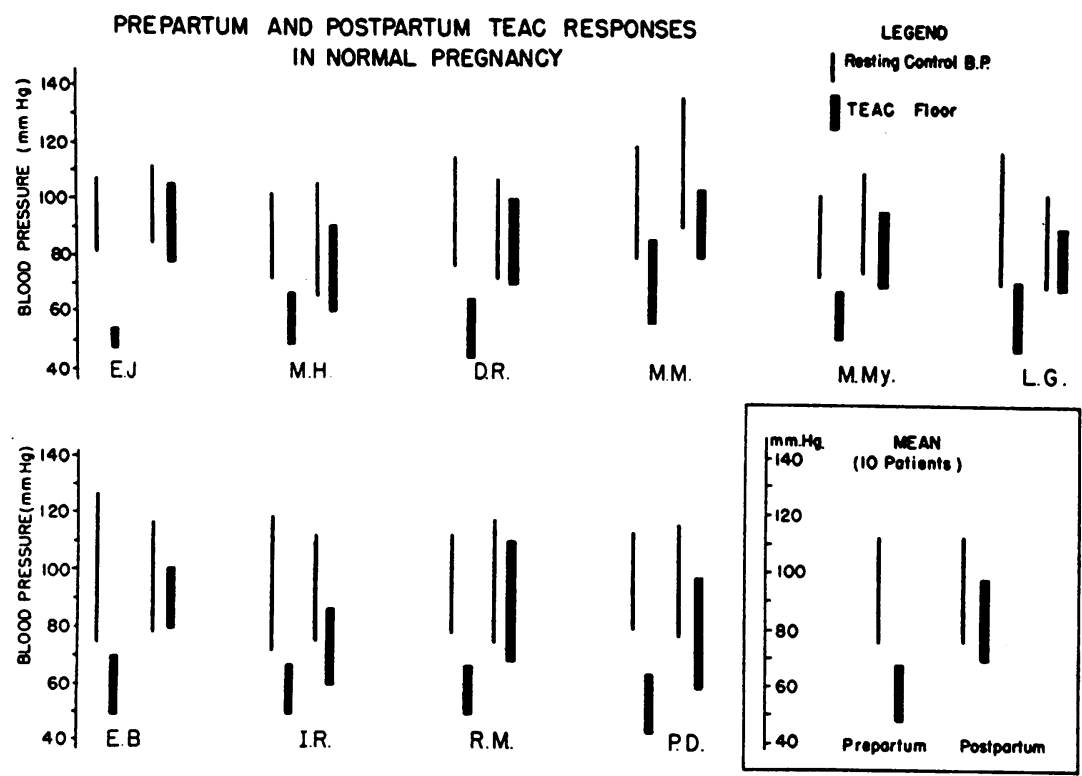

Fig. 2. Prepartum and Postpartum teaC Blood Pressure Responses and Floors in Normal Pregnancy

The responses are grouped in pairs, the first of each pair representing the prepartum response at term and the second the response within 48 hours following delivery. Mean prepartum and postpartum responses for the entire group are shown at the lower right. Note the exceedingly low TEAC floors obtained prepartum and the striking rise to "normal" levels immediately after parturition.

magnitude of these responses is similar to those reported by Lyons in normotensive men and women subjects (1) and to our own observations of young normotensive men (2).

Normal term pregnancy: The results are shown in Figure 2. In each of 10 patients the TEAC floor was strikingly low, ranging from $54 / 44$ to $86 / 56 \mathrm{~mm}$. $\mathrm{Hg}$, with a mean of $68 / 49 \mathrm{~mm}$. $\mathrm{Hg}$. This finding is in striking contrast to the TEAC floors in non-pregnant normotensive females (Figure 1) which ranged from $84 / 56$ to $126 / 80 \mathrm{~mm}$. $\mathrm{Hg}$, the mean being $103 / 67 \mathrm{~mm}$. $\mathrm{Hg}$, although the control blood pressures in both groups were similar. After delivery, the TEAC floor rose to "normal" levels in all patients.

Toxemias of pregnancy: In all 23 cases of toxemia, whether preeclamptic, eclamptic or prepregnant hypertension with superimposed toxemia, the TEAC floor was markedly higher than that found in the group of normal term pregnancy, and fell within the normal range of values upon recovery from toxemia, following delivery or occurring spontaneously. A comparison of the prepar- tum and postpartum TEAC blood pressure floors in toxemia and normal term pregnancy is shown in Figure 3. It is notable that in no instance of toxemia did the diastolic TEAC floor reach levels below $80 \mathrm{~mm}$. $\mathrm{Hg}$, while in normal term pregnancy it did not exceed levels of $56 \mathrm{~mm}$. $\mathrm{Hg}$.

Preeclampsia: The prepartum and postpartum (recovery period) blood pressures and TEAC floors of the 12 patients in this group are shown in Figure 4. During the toxemic phase, there is a moderate though variable blood pressure response to TEAC, the mean control pressure being 159/ $107 \mathrm{~mm} . \mathrm{Hg}$, and the mean TEAC floor 131/94 $\mathrm{mm} . \mathrm{Hg}$. The floor is higher than that of normal non-pregnant females (mean $103 / 67 \mathrm{~mm} . \mathrm{Hg}$ ) and differs strikingly from that of normal term pregnancy (mean $68 / 49 \mathrm{~mm} . \mathrm{Hg}$ ). After delivery and with clinical recovery from the toxemia, the TEAC floor falls consistently to normal levels, the mean floor being $97 / 67 \mathrm{~mm}$. Hg. Comparison of the latter figure to the mean postpartum floor of normal term pregnancy $(98 / 70 \mathrm{~mm} . \mathrm{Hg})$ and to the mean floor for normal non-pregnant females 


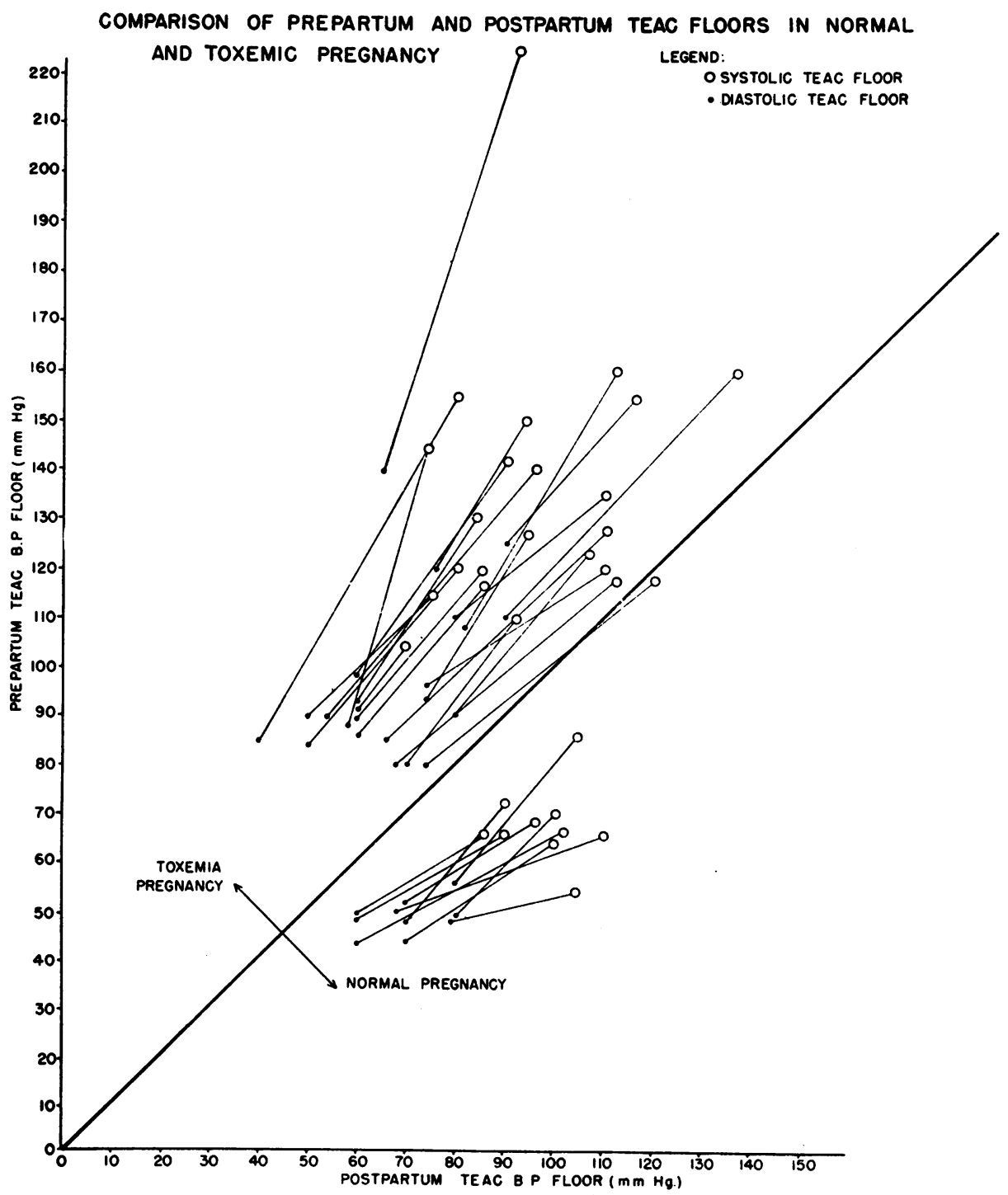

Fig. 3. Comparison of Prepartum and Postpartum teac Blood Pressure Floors in Normal and Toxemic Pregnancy

Systolic and diastolic TEAC blood pressure floors have been plotted against each other in this graph, prepartum studies along the ordinate and postpartum studies along the abscissa. The circles represent systolic floors and the dots diastolic floors. It should be pointed out that if prepartum and postpartum floors were the same, the points plotted would fall on the diagonal. It is obvious that on the basis of blood pressure assay with TEAC all pregnant patients studied fall into two distinct groups, the 23 toxemias above and the 10 normal pregnancies below the diagonal. It can be seen that in those patients above and to the left of the diagonal the postpartum TEAC floor has fallen (toxemias), whereas in those below and to the right of the diagonal, the floor has risen postpartum (normal pregnancy). Note that in toxemia the prepartum diastolic TEAC floor did not descend below $80 \mathrm{~mm}$. $\mathrm{Hg}$, while in normal term pregnancy, the highest prepartum diastolic floor was only $56 \mathrm{~mm}$. $\mathrm{Hg}$. The similarity of systolic and diastolic floors in both groups postpartum should be noted. 
BLOOD PRESSURE IN NORMAL AND TOXEMIC PREGNANCY

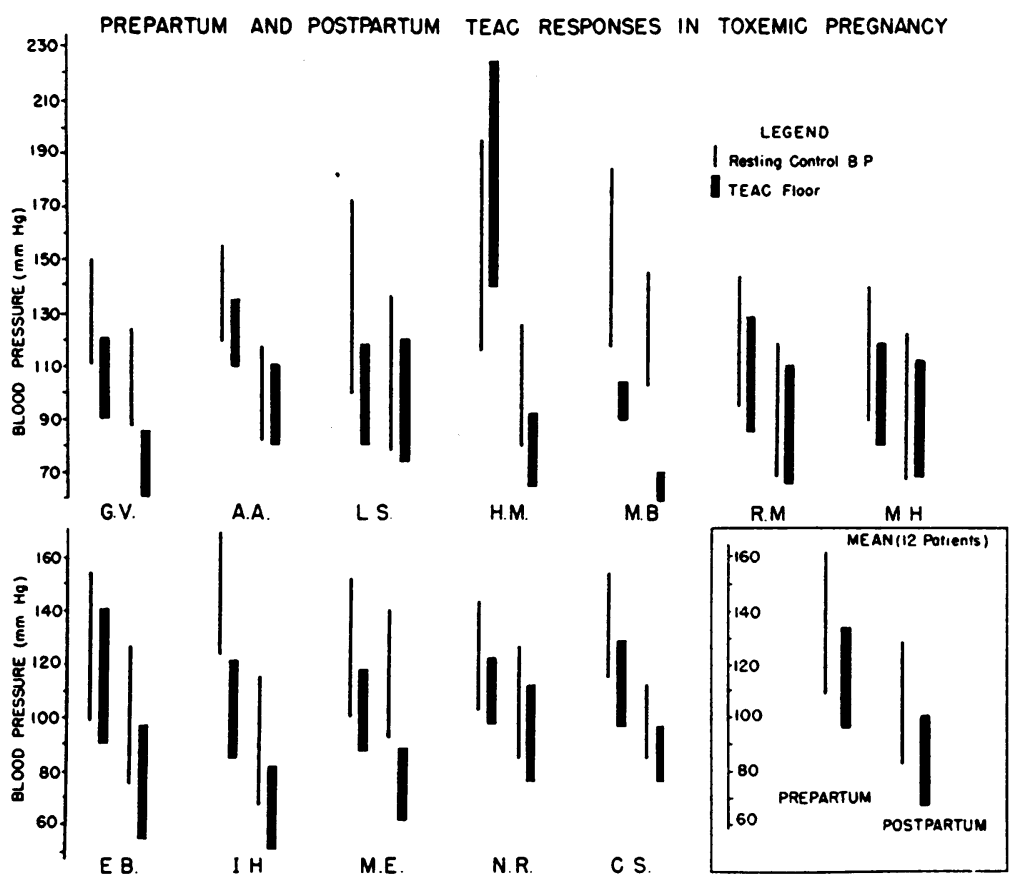

Fig. 4. Prepartum and Postpartum teac Blood Pressure Responses and Floors in Preeclampsia

As in Figure 2, the responses are paired. The first of each pair represents the TEAC response at the height of the toxemia, and the second shows the response coinciding with postpartum recovery from toxemia (between the second and seventh postpartum day in these patients). Rapid descent of the postpartum TEAC floors from abnormally high prepartum (toxemic) test levels is apparent.

$(103 / 67 \mathrm{~mm} . \mathrm{Hg})$ points up the close similarity of these data.

Eclampsia: The results are shown in Figure 5. The TEAC responses and floors in the five cases studied are qualitatively similar to those observed in preeclampsia, but are more striking in that the TEAC floors are higher (mean $135 / 103 \mathrm{~mm} . \mathrm{Hg}$ ). Fall in the floor to the normal range after recovery is more dramatic for this reason (mean $95 / 71 \mathrm{~mm}$. $\mathrm{Hg}$ ).

Essential hypertension with superimposed toxemia: The results are shown in Figure 5. They are similar to those seen in the two groups above. Both the prepartum and postpartum control blood pressures are higher than in the other toxemic groups, and the prepartum TEAC floor is somewhat higher (mean 145/95 mm. $\mathrm{Hg}$ ), although the postpartum TEAC floor is essentially the same (mean 97/67 mm. Hg).

The mean TEAC blood pressure responses and floors are compared graphically in Figure 6. For this purpose all patients with toxemia have been considered as a single group, whether preeclamptic, eclamptic or prepregnant hypertension with superimposed toxemia. Prepartum and postpartum studies are compared with those of normal pregnancy and with responses of non-pregnant controls. The mean prepartum TEAC floor of all toxemias is $135 / 96 \mathrm{~mm}$. $\mathrm{Hg}$, and descends to $97 /$ $67 \mathrm{~mm}$. $\mathrm{Hg}$ with postpartum recovery.

Changes in the TEAC floor and blood pressure response during fuctuations in the severity of toxemia

Figure 7 illustrates the changing responses and floors in four patients whose clinical courses showed fairly marked fluctuation in both the antepartum and puerperal periods. The TEAC floor consistently was observed to rise and fall in relation to exacerbations and remissions of the toxemic state. These fluctuations in TEAC floor were re- 


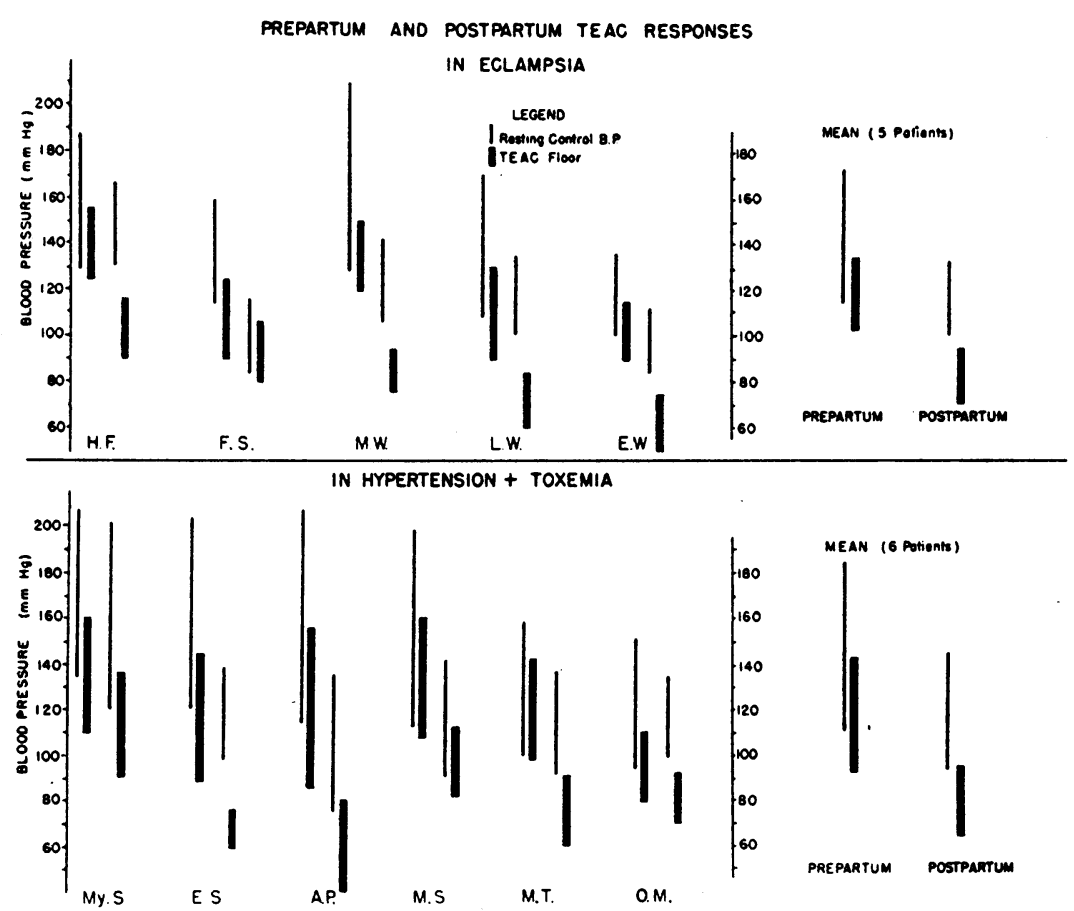

Fig. 5. Prepartum and Postpartum teaC Blood Pressure Responses and Floors in Eclampsia and Hypertension with SUperimposed Toxemia

As in Figure 4, the second of the paired responses was obtained postpartum during the phase of recovery from toxemia. As in preeclampsia, the TEAC floors are abnormally elevated at the height of the toxemia and descend rapidly to "normal" levels during the recovery period from toxemia following delivery.

peatedly noted to be independent of the height of the control blood pressure.

\section{DISCUSSION}

Acheson and Moe (3) in animals and Lyons, et al. (4) in man have demonstrated that TEAC produces a transient blockade of the autonomic nervous system at the ganglionic level, and that the resulting fall in blood pressure is due primarily to a decrease in peripheral resistance (arteriolar tonus). In addition it has been shown that pressor agents (angiotonin, epinephrine) which act directly on the blood vessels are capable of producing pressor responses when autonomic impulse transmission has been completely eliminated by TEAC (5).

Evidence for the completeness of the sympathetic blockade induced by acute intravenous doses of $400 \mathrm{mg}$. of TEAC is presented in detail elsewhere (6). A brief summary of the pertinent information is appropriate here: $(a)$ the TEAC floor parallels but is consistently lower than the blood pressure floor induced by high spinal anesthesia to T-3 levels; $(b)$ the TEAC floor is not lowered further by doubling the dose in the same individuals; and (c) TEAC abolishes the minute cold pressor blood pressure effect, a response mediated through the sympathetic nervous system (7). Thus, it may be concluded that the blockade achieved is sufficiently complete for the purposes of this investigation, since a constant dose was employed in all groups studied and in the same individuals under the varying conditions of study.

That the sympathetic nervous system mediates active vasomotor control of the arteriolar bed is now well established. In this clinical assay of responses following autonomic blockade with TEAC, the active neurogenic component of blood pressure maintenance may be assumed to have been eliminated, and the resulting blood pressure (floor) is therefore maintained by humoral and intrinsic mechanisms. 
COMPARISON OF MEANS.

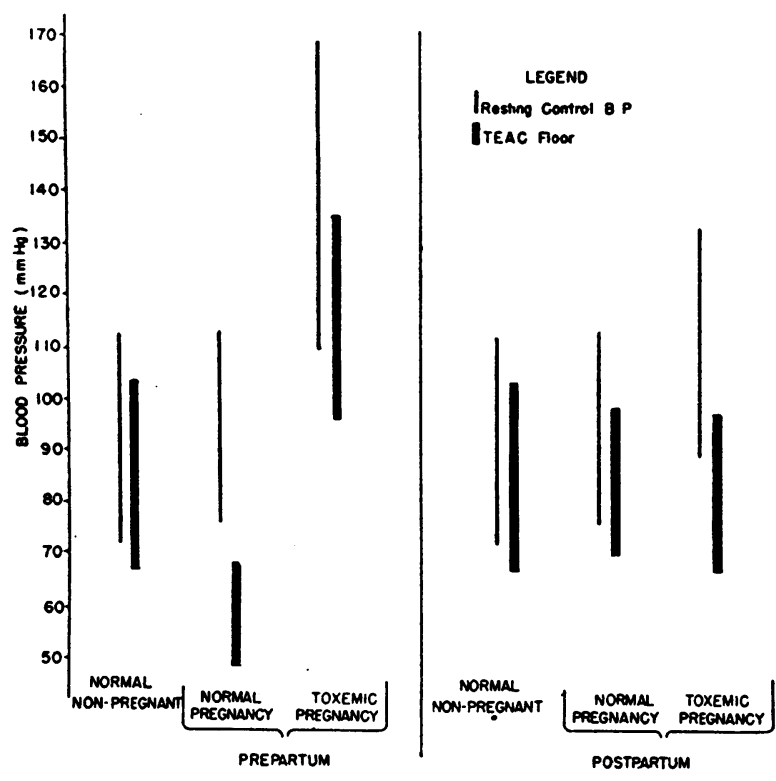

Fig. 6. Mean Prepartum and Postpartum TeaC Blood Pressure Responses of Normal Term Pregnancy and Combined Toxemia Groups

For comparison purposes the mean response of normal non-pregnant controls has been included here. The toxemia mean includes all 23 cases studied, whether preeclamptic, eclamptic or pre-pregnant hypertension with toxemia. Postpartum shift of the TEAC floors of the pregnant groups to levels exactly corresponding to that of the non-pregnant controls should be noted.

It is obvious from Figure 1 that normal nonpregnant women are, generally speaking, poor responders to TEAC. Despite slight variations from individual to individual, the mean blood pressure fall of $10 / 5 \mathrm{~mm}$. $\mathrm{Hg}$ reflects the minor role played by sympathetic vasoconstrictor tone in maintaining blood pressure in non-pregnant normals.

Rapid shifting of postpartum TEAC floors (rising floors in normal pregnancy, falling floors in toxemia) suggests a striking difference in the mechanism of blood pressure maintenance at term of normal pregnancy from that involved in sustaining the hypertension of toxemia. Blood pressures in prepartum term normals invariably fell to exceedingly low levels with sympathetic blockade, indicating neurogenic domination in this group. Release of sympathetic tone with TEAC in toxemia caused varying degrees of blood pressure fall, but diastolic TEAC floors rarely descended below $90 \mathrm{~mm}$. $\mathrm{Hg}$, suggesting activity of humoral mechanisms in sustaining hypertension in these patients.

The fall in blood pressure induced by TEAC in man $(1,8)$ is not associated with relevant changes in cardiac output or blood volume. However, term pregnancy is attended by blood volume alterations and by venous pooling in the lower extremities due to the increased venous pressure in the tributaries to the inferior vena cava which results from the pressure of the gravid uterus. Therefore, consideration must be given these changes as possible factors influencing the shifts observed in the TEAC response and floor in the pregnant patients studied. In toxemia the plasma volume is either normal or decreased (9), whereas it is regularly increased in normal term pregnancy (10). Since the levels of both the control blood pressures and TEAC floors are diametrically opposite to what might be expected from the blood volume shifts after delivery, this possibility is eliminated as a cause for the divergent floors.

Likewise, since the factors which increase the venous pressure in the lower extremities are essentially the same in toxemia and normal term pregnancy, it is probable that the venous pressure is similarly elevated in both conditions, returning to normal after delivery. Therefore, the opposite effects of TEAC in normal and toxemic pregnancy cannot be due primarily to differences in venous pressure.

Since TEAC causes transient postural hypotension, perhaps through lessening neurogenic venous tone, it is quite possible that under conditions of term pregnancy, when the venous pressure in the lower extremities is elevated, release of venous tone may exaggerate venous pooling and, therefore, contribute to the striking fall in blood pressure which occurs in normal term pregnancy. Regardless of the relative roles of arteriolar and venous tone in the blood pressure response to TEAC in normal term pregnancy, it is most probable that factors other than neurogenic tone, namely, humoral factors, contribute to the elevated TEAC blood pressure floor in toxemia. The relative roles of venous pooling and arteriolar tone in the blood pressure response to TEAC in pregnancy are being further investigated.

The contention that the extent of the decrease in pressure is largely dependent on the initial elevation of pressure is untenable here. Many of the 


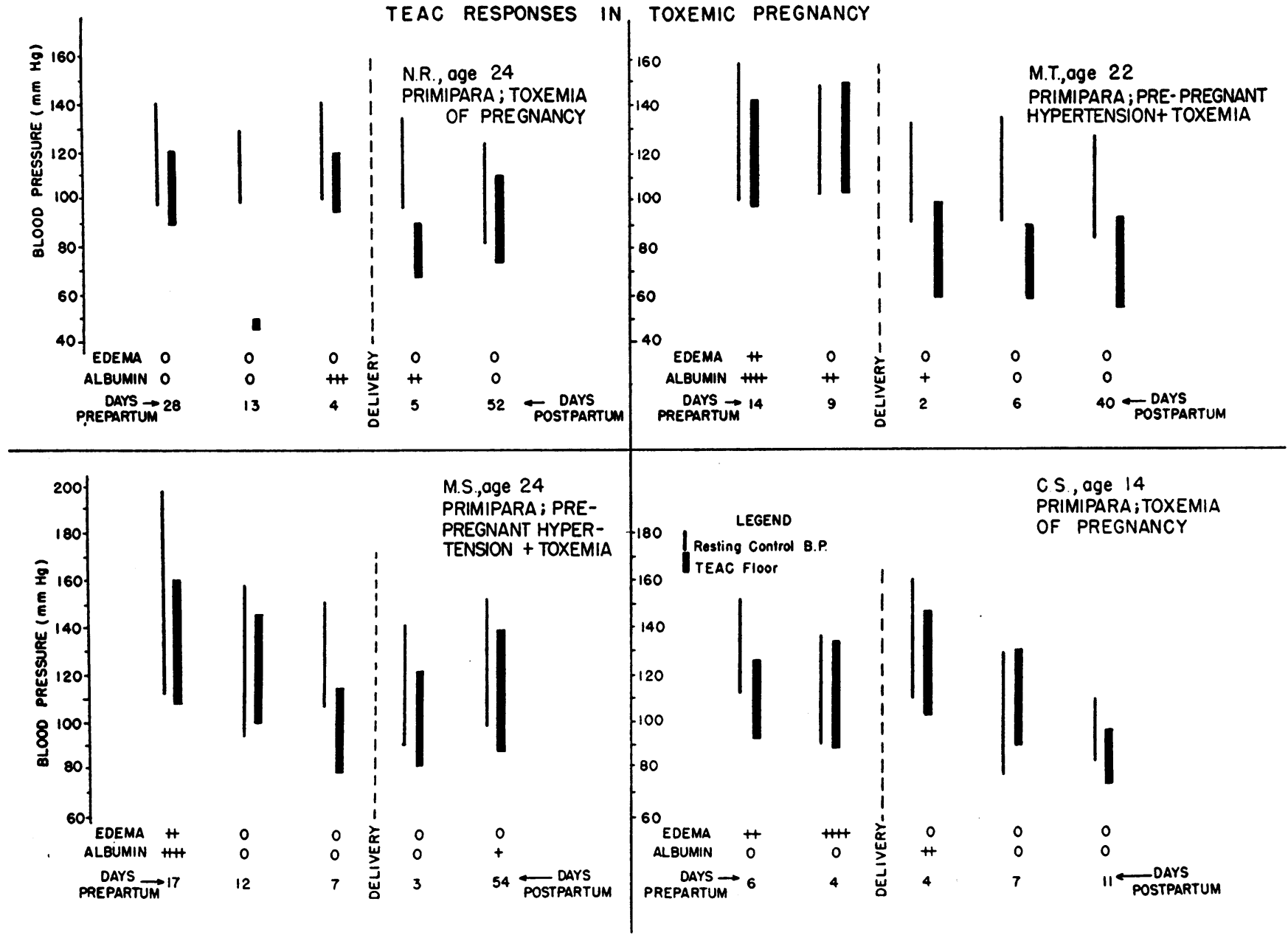

Fig. 7. Changes in the teaC Blood Pressure Response and Floor during Fluctuations in the Severity of Toxemia

Pictured are the responses of four representative toxemia patients who were tested repeatedly during prepartum and postpartum periods in an effort to correlate TEAC responses and floors with fluctuations in the severity of toxemia.

Patient N. R. was considered toxemic 28 days prepartum, because of progressive rise in blood pressure to persistent hypertensive levels observed in the course of prenatal visits. Following hospitalization, bedrest and restricted salt diet, the blood pressure returned to normal and the patient was discharged. Thirteen days prepartum she was totally free of signs and symptoms of toxemia and a TEAC response typical of normal term pregnancy was obtained. Hypertension and albuminuria appeared one week prepartum and on the fourth day prior to delivery the TEAC response and floor conformed to the toxemic pattern.

Patient M. T. illustrates a case in which the toxemic symptomatology and toxemic blood pressure responses to TEAC persisted until delivery.

Patient M. S., a prepregnant hypertensive with superimposed toxemia, showed marked prepartum remission of toxemia. The TEAC blood pressure floors likewise showed improvement (descended) simultaneous with the clinical improvement although responses similar to those of normal term pregnancy were not obtained.

Patient C. S. exhibited severe edema and moderate hypertension in the antepartum period. TEAC floors were consistently elevated. Marked exacerbation of hypertension and appearance of albuminuria followed delivery, and the TEAC floor rose still higher. With recovery from toxemia, the TEAC floors gradually returned to normal. 
eclamptics and pre-pregnant hypertensives persisted with elevated blood pressure following the disappearance of other toxic signs. Despite this, the TEAC floors invariably descended during the puerperium. The results in normal pregnancy further refute this concept by consistently showing rising TEAC floors immediately postpartum even though initial pressures were identical with prepartum levels.

Thus, the data presented offer physiologic support for the humoral theory of pregnancy toxemia so far as the hypertension is concerned. Simultaneously, attention is directed to the nature of alteration of blood pressure control in normal pregnancy. The evidence suggests that the blood pressure of the normal non-pregnant female is supported largely by humoral (plus intrinsic) tone. Although the pressure remains normal or low during normal pregnancy, at some time during gestation the humoral component is relegated to a minor role, and neurogenic (plus intrinsic) tone predominates at term. Immediately postpartum, humoral factors again come into play presumably to the same degree as in control normotensive subjects. When toxemia develops in the course of pregnancy, humoral mechanisms support a considerable portion of the associated blood pressure elevation. Nevertheless, toxemic blood pressures are frequently reduced following sympathetic blockade, indicating that neurogenic mechanisms are still operative. Whether the blood pressure response to TEAC is due to release of some remaining neurogenic arteriolar or venous tone or to a combination of the two, remains to be determined.

Research in pregnancy toxemia recently has centered about the search for increased quantities of humoral agents and deficiencies of inhibitor substances. Page has found plasma angiotonase levels elevated four to ten times in normal pregnancy (11). This finding is of increasing interest in view of the evidence for diminished humoral tone demonstrated in normal term pregnancies in the data just presented. Histaminase and pitocinase are likewise elevated in normal pregnancy. Deficiencies of progesterone and estrogens and increased amounts of chorionic gonadotropin have been described in toxemia. Interestingly, the temporal relationships of rising and falling titers reported for many of these substances (12) corre- spond closely to the rising and falling of the TEAC floors before and after delivery.

There is considerable evidence that clinical assay with TEAC may offer valuable assistance in the diagnosis of toxemia and in following the course and severity of the disorder. Figure 7 shows how, in general, the height of the TEAC floor parallels the severity of the toxemia. The highest diastolic floors observed occurred in two patients with eclampsia and in the most severe case of preeclampsia studied. Cases which fluctuated in severity showed corresponding fluctuation in the height of the TEAC floor.

From the standpoint of diagnosis there is as yet no evidence that TEAC assay can differentiate between toxemia and essential hypertension uninfluenced by pregnancy. The greatest diagnostic assistance offered is in those pregnant patients admitted with borderline elevations of blood pressure. On the basis of the current study, it may be stated that a patient who experiences a TEAC response resulting in a diastolic blood pressure floor considerably below $90 \mathrm{~mm}$. $\mathrm{Hg}$ would be unlikely to have toxemia.

\section{CONCLUSIONS}

1. A comparative study has been made of the TEAC blood pressure response during and after recovery from toxemia of pregnancy, in the prepartum and postpartum periods of normal term pregnancy, and in normal non-pregnant women.

2. In normal term pregnancy the TEAC blood pressure floor is strikingly low and rises to normal levels after delivery. In toxemia the TEAC floor is higher than normal and consistently falls to normal levels after recovery.

3. Since TEAC, by blocking the autonomic nervous system at the ganglia, eliminates neurogenic tone but does not lessen humoral tone, the results suggest that the hypertension of toxemia of pregnancy is supported by an excessive degree of humoral tone.

4. Because of the striking depressor response to TEAC in normal term pregnancy, it appears likely that neurogenic mechanisms are more active in normal pregnancy than in toxemia.

5. Clinical assay with TEAC may be a helpful aid in diagnosis of toxemia of pregnancy and in the evaluation of changes in severity during its course. 


\section{BIBLIOGRAPHY}

1. Lyons, R. H., Hoobler, S. W., Neligh, R. B., Moe, G. K., and Peet, M. M., Experiences with tetraethylammonium chloride in hypertension. J. A. M. A:, 1948, 136, 608.

2. Unpublished data.

3. Acheson, G. H., and Moe, G. K., Some effects of tetraethylammonium on the mammalian heart. J. Pharmacol. \& Exper. Therap., 1945, 84, 189; The action of tetraethylammonium on the mammalian circulation. Ibid., 1946, 87, 220.

4. Lyons, R. H., Campbell, K. N., Moe, G. K., Neligh, R. B., Hoobler, S. W., Berry, R. L., and Rennick, B. $k$., The effects of blockade of the autonomic ganglia in man with tetraethylammonium. Am. J. M. Sc., 1947, 213, 315.

5. Moe, G. K., Rennick, B. R., Hoobler, S. W., Neligh, R. B., and Lyons, R. H., The evaluation of vasomotor tone in animals and man by means of the tetraethylammonium ion. Proc. Central Soc. Clin. Research, 1946, 19, 5.

6. Ferris, E. B., Jr., Reiser, M. F., Stead, W. W., and Brust, A. A., Clinical and physiologic interrela- tionships in arterial hypertension. Tr. A. of Am. Physicians (In press).

7. Reiser, M. F., and Ferris, E. B., The nature of the cold pressor test and its significance in relation to neurogenic and humoral mechanisms in hypertension. J. Clin. Invest., 1948, 27, 156.

8. Stead, W. W., Reiser, M. F., Rapoport, S., and Ferris, E. B., The effect of sodium chloride depletion on blood pressure and tetraethylammonium chloride response in hypertension. J. Clin. Invest., 1948, 27, 766.

9. Freis, E. D., and Kenny, J. F., Plasma volume, total circulating protein and "available fluid" abnormalities in preeclampsia and eclampsia. J. Clin. Invest., 1948, 27, 283.

10. McLennan, C. E., and Thouin, L. G., Blood volume in pregnancy. Am. J. Obst. \& Gynec., 1948, 55, 189.

11. Page, E. W., Plasma angiotonase concentration in normal and toxemic pregnancies. Am. J. M. Sc., 1947, 213, 715.

12. Smith, G. V., and Smith, O. W., Internal secretions and toxemia of late pregnancy. Physiol. Rev., 1948, 28, 1. 